Bond University

Research Repository

\title{
Financial Applications of Semidefinite Programming: A Review and Call for Interdisciplinary Research
}

Gepp, Adrian; Harris, Geoffrey; Vanstone, Bruce J

Published in:

Accounting and Finance

DOI:

10.1111/acfi. 12543

Licence:

Other

Link to output in Bond University research repository.

Recommended citation(APA):

Gepp, A., Harris, G., \& Vanstone, B. J. (2020). Financial Applications of Semidefinite Programming: A Review and Call for Interdisciplinary Research. Accounting and Finance, 60(4), 3527-3555.

https://doi.org/10.1111/acfi.12543

\section{General rights}

Copyright and moral rights for the publications made accessible in the public portal are retained by the authors and/or other copyright owners and it is a condition of accessing publications that users recognise and abide by the legal requirements associated with these rights.

For more information, or if you believe that this document breaches copyright, please contact the Bond University research repository coordinator. 


\title{
Financial Applications of Semidefinite Programming: A Review and Call for Interdisciplinary Research
}

\begin{abstract}
Optimization problems in finance commonly have non-linear constraints for which previous solutions have required unrealistic assumptions. However, many of these can be efficiently solved as Semidefinite Programming (SDP) problems, which have less restrictive assumptions. Through review of the literature that uses SDP in finance, two major research streams are identified: portfolio optimization and option pricing. Nevertheless, many finance researchers are unaware of SDP. One possible reason is that this research is often published in non-finance journals. This paper aims to better integrate the SDP research to promote wider use of current findings and further interdisciplinary research, particularly in environmental finance.
\end{abstract}

Key Words: Semidefinite Programming, Optimization, Portfolio, Option Pricing, Environmental Finance

JEL Codes: C61, G11, G12 


\section{Introduction}

There are numerous optimization problems in finance such as portfolio asset allocation, option pricing, risk management and equilibrium analysis. These problems commonly have nonlinear constraints for which previous solutions have required strong, often linear, assumptions in order to find solutions. Traditional methods for solving these problems abound and are diverse recent examples include modelling asymmetric dependence in portfolio formulation using copula functions (Low, 2017; Hartherley and Alcock, 2007), pricing options allowing for stochastic volatility utilising Lévy processes ( $\mathrm{Li}$ et al., 2017), analysing foreign equity bias in mutual funds using Bayesian and other approaches (Mishra, 2016), and portfolio optimization in the presence of transactions costs (Suh, 2016). Smith and Walsh (2013) provide an important review of the CAPM approach that underpins much of the portfolio optimization research, highlighting the important implication that there is an infinite number of ex post efficient portfolios.

Many of these optimization problems can be cast as SDP problems, which have less restrictive assumptions while still being able to be solved efficiently. By reviewing the literature that uses SDP in finance, two major research streams are identified: portfolio optimization and option pricing.

There are an abundance of problems in finance that can be characterized as optimization under uncertainty. These problems are known mathematically to be NP-hard problems and include examples such as maximizing portfolio returns subject to specified constraints, optimal multiperiod investment strategies subject to given conditions (such as cardinality constraints), as well as computing bounds on options prices. In option pricing, SDP may also be used as an alternative to the classic Black-Scholes framework (Kwon and Li, 2016). In the research reviewed below, SDP has been used to either solve problems that traditional finance methods cannot, or to extend the mathematical limitations of existing approaches to produce near optimal solutions in a reasonable computation timeframe.

Semidefinite programming (SDP) is less commonly known as semidefinite optimization (SDO) and is essentially an extension of linear programming (LP). SDP has been studied as far back as the 1940s under various names, such as linear matrix inequalities (LMIs) and linear optimization with matrix variables (Wolkowicz et al., 2000). The study of the cone of semidefinite matrices even predates LP (Pardalos and Wolkowicz, 1998a). However, because of the lack of efficient solution algorithms SDP has had a slow and scattered history unlike the rapid growth experienced by LP. The discovery of efficient algorithms to solve SDP problems, coupled with their diverse range of application areas, was the major factor behind the growth of SDP in the 1990's (Helmberg, 2000). Although SDP is a growing field, LP models are still more widely used than SDP models.

Even though SDP has been considered a standard optimization tool in some fields for many years, it still remains largely unknown to finance researchers. A major cause of this is believed to be a lack of introductory papers, and this paper helps address that gap.

The objectives of this paper, which are addressed in order, are to

- provide an introduction and brief review of the general field of SDP for a finance audience. 
- $\quad$ Present an overview of the algorithms and software to solve SDP problems.

- $\quad$ Review the research that applies SDP to financial optimization problems.

Although this paper is directed at finance experts who are not familiar with SDP, it does require the reader to be somewhat familiar with linear algebra. Appendix A provides a brief overview of the underlying concepts in SDP. When setting up a finance problem to be solved via SDP, one needs to establish the optimisation function and the semi-positive definite constraints. For specific references, Appendix B demonstrates how the problem was formulated within an SDP framework.

\section{Introduction to SDP}

SDP involves optimizing a linear objective function subject to linear constraints, where the objective function is optimized over the closed convex cone of semidefinite matrices. The general form for an SDP problem is:

Minimize the objective function $C \cdot X$ subject to the constraints

$A_{i} \bullet X=b_{i}$ for $i=1, \ldots, m$

and the positive semidefinite constraint $X \geqslant 0$.

where $X$ is a $n \times n$ variable matrix of the free parameters, $C$ and the $A_{i}{ }^{\prime} s$ are given $n \times n$ symmetric matrices ${ }^{1}$ of problem-specific real coefficients, and the $b_{i}{ }^{\prime} s$ are given problem-specific real scalars and $m$ is the number of linear equality constraints.

Interestingly, if all the given matrices $\left(C, X, A_{i}{ }^{\prime} S\right)$ in an SDP problem were diagonal then the SDP problem would reduce to an LP problem, where the variable vector $x$ is formed by the diagonal of $X$ (Freund, 2004; Wright, 2004). Consequently, it is not surprising that many parallels exist between LP and SDP (Wolkowicz et al., 2000). However, SDP possesses some subtle, yet interesting differences and is far more generalizable than LP. SDP can solve many integer LP and non-linear optimization problems efficiently. SDP encompasses LP, quadratic programming (QP), quadratically constrained quadratic programming (QCQP) and second-order cone programming (SOCP) as special cases (de Klerk, 2002; Wolkowicz, 2002). Thus, SDP offers a common way to represent these convex optimization problems, which allows unified analysis of properties and solution algorithms (Vandenberghe and Boyd, 1996).

\subsection{Duality for SDP problems}

Duality is the concept that an optimization problem can be viewed as either a primal problem or dual problem (Vandenberghe and Boyd, 1996). This ability to view problems in different ways can assist in finding solutions, particularly for SDP. First recognised by Bellman and Fan (1963), much of the theory of duality extends from LP to SDP. The differences between LP and SDP duality are detailed by Ramana (1997) and Ramana et al. (1997). SDP has an elegant theory of duality very similar to LP, but the theory is decidedly more complex as the cone of semidefinite matrices is not polyhedral like the feasible region of an LP problem (Helmberg,

\footnotetext{
${ }^{1}$ Since $X$ is symmetric there is no loss in generality in assuming that $C$ and the $A_{i}{ }^{\prime} s$ are symmetric (Freund, 2004).
} 
2000; Wright, 2004). As with LP it is easy to switch between the primal and dual ${ }^{2}$ forms of SDP problems.

The primal form of the SDP problem refers to the original SDP problem, which can be stated in various ways. Similarly, there are various forms in which the dual problem can be stated; most commonly, the dual problem is defined as

Maximise the objective function

$$
\sum_{i=1}^{m} b_{i} y_{i} \quad \text { where } y \in \mathbb{R}^{n} \quad(\text { or alternatively written as } B \cdot Y \text { ) }
$$

subject to the constraint

$$
\begin{aligned}
& S+\sum_{i=1}^{m} y_{i} A_{i}=C \\
& \text { and the positive semidefinite constraint } S \geqslant 0 .
\end{aligned}
$$

That is, its dual is a maximization problem where the matrix $C-\sum_{i=1}^{m} y_{i} A_{i}$ must be positive semidefinite. Hence, the dual problem is also an SDP problem. SDP duality is weaker than that of LP (Freund (2004) and Wright (2004) present a proof of this) as strong duality only holds under certain constraints. This weak duality does not guarantee that there is a dual equivalent for every primal problem (and vice-versa) nor that the duality gap is zero at optimality, but it is still known that

- The duality gap is nonnegative at all feasible solutions $(C \bullet X-B \bullet Y \geq 0)$

- If the duality gap is zero then optimality has been achieved, but a non-zero duality gap is possible at optimality (duality gap $=0 \Rightarrow$ optimality).

The SDP dual is often more practically useful; it is often easier to handle as the variables in the constraints and the objective function are the same (Freund, 2004). This is different from LP, where the primal LP problem is preferred to the dual in most practical situations. For more information on duality in SDP, the summary paper by Vandenberghe and Boyd (1996) contains a detailed introduction.

\section{Algorithms to Solve SDP problems}

The semidefinite constraint is a non-linear convex constraint. In fact, Freund (2004) presents an elegant proof that the set of semidefinite matrices is a closed convex cone (see Appendix A.3) within the set of symmetric matrices. The ramifications of this are that the feasible region of an SDP problem is convex which in turn means that there is one optimal point on the boundary of the feasible region.

The convex feasible region of an SDP problem may contain curves, which means that the basic feasible solution (BFS) comprises the finite number of vertices as well as an infinite number

\footnotetext{
2 To be precise, we are referring to the Lagrangian dual throughout this section.
} 
of points along all curves. Consequently there is no simple or practical Simplex algorithm for SDP. It is also not yet possible to determine (in)feasibility of an SDP problem in a finite number of iterations (tests). Nevertheless, exact complexity bounds have been found by Porkolab and Khachiyan $(1997,1998)$ who show that many SDP problems are solvable (or determined to be infeasible) in polynomial time. Furthermore, problems that are not exactly solvable in polynomialtime can be solved to a predefined level of precision in polynomial-time (Grötschel et al., 1988; Wolkowicz, 2002). Grötschel et al. (1988) used the ellipsoid algorithm to show that SDP problems can be solved to any fixed precision in polynomial time, but again the algorithm is practically inefficient and more efficient Interior-Point (IP) algorithms are preferred (Laurent and Rendl, 2002; Vandenberghe and Boyd, 1996; Wolkowicz, 2002).

These algorithms for solving SDP problems (including discrete versions) are discussed in more detail in the following subsections, along with references to software packages that implement SDP algorithms.

\subsection{Interior-Point (IP) Algorithms}

The breakthrough for the SDP field came when Nesterov and Nemirovskii (1988, 1990a,b, 1994) presented efficient polynomial-time, IP algorithms for general convex optimization problems and then specifically for SDP problems and was a major reason for its growth in popularity. Independently, Alizadeh $(1991,1992)$ and Kamath and Karmarkar $(1992,1993)$, also generalized the existing LP interior-point algorithms to SDP.

IP algorithms search for the optimal solution by going through the middle of the feasible region, rather than around the edge as the Simplex algorithm does. The algorithm essentially follows a smooth curve through the feasible region, commonly known as the central path, by using the Newton method. The central path passes through the analytic centre of the feasible region and converges upon the optimum point. Vandenberghe and Boyd (1996), Helmberg (2000, section 4.1) and Gärtner and Matoŭsek (2012, p. 99-118) provide excellent treatments of the concepts of analytic center and central path, which are based upon barrier functions. When using IP algorithms, the optimal point is identified by a duality gap of zero, and thus IP algorithms assume strong duality. The importance of duality in these IP algorithms is the reason they are more accurately called primal-dual IP algorithms. It is also assumed that the constraints are linearly independent. For more information, Wright (2004), Laurent and Rendl (2002) and Freund (2004) also provide excellent introductions to IP algorithms in SDP.

\subsection{Algorithms for Discrete Problems}

In the same way cutting plane algorithms can be used in LP to solve integer IP problems (see Appendix A.6), cutting plane algorithms have more recently been explored to solve some SDP problems with discrete variable constraints. Cutting plane algorithms, based on IP algorithms, are presented by Helmberg and Weismantel (1998), who use the max-cut and quadratic 0-1 knapsack problems as examples. However, the IP algorithm's computation time dramatically increases with the number of cutting planes added, as each additional inequality increases the computation time of the IP algorithm. Thus, IP cutting plane algorithms are limited 
to small problems. This is the main reason given by Helmberg (2000) for the rarity of cutting plane techniques in SDP applications, despite their positive theoretical results for practically useful problems.

\subsection{Software to Solve SDP}

SDP solvers are available in a variety of software packages and many of them are freely available. The increasingly popular, open source $\mathrm{R}$ programming language offers interfaces with multiple SDP solvers. Rcsdp (Bravo, 2016) and Rdsdp (Zhu and Ye, 2016) offer interfaces to two open source SDP solvers, CSDP (Borchers, 1999b) and DSDP (Benson et al., 2000) respectively. Both SDP solvers use IP algorithms on both primal and dual problems, and both take advantage of sparsity. A user guide is available for CSDP (Borchers, 1999a), which has a variant that is able to exploit multiple CPUs/cores for parallel computing (Borchers and Young, 2007). SDPA (Yamashita et al., 2012) is an open source alternative that uses an IP algorithm. While SDPA is written in $\mathrm{C}++$, program variants are available that offer interfaces for Matlab and Python, as well as for enabling parallel computing.

In contrast to the more popular IP algorithms, the open source Splitting Conic Solver (SCS) (O’Donoghue et al., 2016a,b) uses a first-order algorithm for solving SDP and other optimization problems. As mentioned above, this approach scales to extremely large optimization problems better than IP algorithms. Inferfaces for SCS are available in R, as well as Python, Java, C, C++, Matlab, Julia and Scala.

A commercial alternative is MOSEK, which can be used for a variety of linear and nonlinear optimization problems including SDP. MOSEK offers multiple algorithms to solve these problems, most notably IP algorithms. It also detects infeasible solutions, supports parallel computing and comes with all the support typically expected of commercial products. MOSEK can be accessed from a variety of interfaces including $\mathrm{C}, \mathrm{C}++$, Java, .NET, MATLAB, Python and R. Koenker and Mizera (2014) provide an introduction to convex optimization in R including the Rmosek package that interfaces with MOSEK. Although it is a commercial product, it is important to note that academic licences for research and education are available at no cost.

\section{SDP in Finance}

SDP has been successfully used to solve a variety of finance-specific problems. There are many optimization problems in finance that have non-linear constraints, for which previous solutions have required strong assumptions in order to find solutions. Consequently, it is desirable to find semidefinite relaxations for these problems to avoid these unrealistic assumptions while still being able to solve the problems efficiently.

A systematic approach has been followed to collect publications for inclusion in this review of SDP in finance. First, the Scopus database was used to search the title, abstract and keywords of publications for those that contained both [('semidefinite' AND 'programming') OR 'sdp'] and 
('finance' OR 'portfolio') $)^{3}$. This initial search returned many engineering articles and so the results were filtered to exclude the subject area 'engi*'. A total of 50 papers resulted as of October 2017. Next, articles that merely mentioned the potential for financial applications of SDP without actually addressing them were removed and additional references were added by searches of cited references, ISI Web of Science and Google Scholar. The process resulted in a total of 36 journal and 2 conference publications. Working papers, technical reports and textbook chapters were also identified and are cited in future sections, but these are not included in the following summary analysis.

The vast majority of publications are in Operations Research or Optimization outlets, as shown in Figure 1. Given that finance academics are less likely to follow these two bodies of literature, this is one possible reason for SDP being relatively unknown in finance. Furthermore, although Finance outlets account for the same number of publications as Optimization outlets, the majority of publications in Finance outlets are older; of the 20 'SDP in finance' publications from 2012 onwards, only one has been in a Finance outlet. This review paper contributes to bridging the gap between these two bodies of literature by making the findings more accessible to finance academics.

[insert Figure 1 approximately here]

[insert Table 1 approximately here]

An analysis of the citation information from Scopus reveals three top publications; no others had either more than 100 citations or an average greater than seven per year. The top papers are listed in Table 1 and it is interesting to note than none of them are published in Finance outlets.

The publications were also categorized by the authors according to their main research focus. This process revealed two main research streams being portfolio optimization and option pricing, which will be discussed in the next two subsections. Following that, the one publication that did not fall into either stream is discussed, before future research opportunities are identified.

\subsection{Portfolio Optimization}

Portfolio optimization involves allocating capital over a set of available assets to maximize return while minimizing risk. Portfolio optimization problems involving VaR are well known to be typically computationally intractable and the complexity becomes NP-hard when the portfolio contains derivatives (Zymler et al., 2013). This task is further complicated by the many available measures of risk. The best known mathematical model and the most famous portfolio optimization approach, now known as modern portfolio theory, was developed by Markowitz (1952, 1959). Markowitz's work brought about important advances in the asset allocation and asset pricing fields of finance. This approach has since been extended to tracking-error optimization (Roll,

\footnotetext{
${ }^{3}$ The term portfolio was included because, in the authors' experience, papers in this area of finance do not always use the term finance prominently.
} 
1992), as the success of professional portfolio managers is commonly determined by the excess of their portfolio returns over a specified benchmark portfolio.

Markowitz's model is a quadratic programming problem, which is also referred to as the mean-variance model, because the variance of portfolio returns is used as the measure of risk. Despite the strong theoretical contribution of the Markowitz Mean-Variance model (MMV) it has been highly criticized in practice. The main disadvantage of the model stems from the high sensitivity to its parameters, especially the expected return (mean) of individual assets. Parameters such as the expected return of assets are usually estimated from noisy historical data, and consequently cannot be estimated with precision. This concern provides the impetus for studying techniques that consider both the uncertainty modelled by the parameters and the uncertainty of the parameters themselves.

Many techniques have been suggested to reduce the parameter sensitivity of portfolio optimization models; Goldfarb and Iyengar (2003) provide a list of these approaches including references to relevant research. Robust versions of the portfolio optimization problem acknowledge the uncertainty of the input parameters and aim to systematically combat the sensitivity of the optimal portfolio to errors in the estimates of the inputs ${ }^{4}$. This is done by imposing the model's constraints over the set of plausible values of the parameters, rather than the single most likely value. The new robust optimization problem is then solved assuming the worstcase behaviour within the plausible set of the parameter values (Lutgens and Sturm, 2002).

Costa and Paiva (2002) addressed the tracking-error extension of the robust portfolio optimization problem, using variance as the measure of risk. They showed that the error tracking formulation problems were SDP problems. Their empirical testing on a small amount of São Paulo Stock Exchange data revealed that using SDP is useful, especially when minimizing the amount of future portfolio re-balancing and transaction costs are important.

Since these early published works involving recasting portfolio optimization into a framework that can be solved using SDP there has been a steadily increasing number of publications in this area. The later publications have successfully extended both aspects of recasting the optimization problem as well as introducing further complexity into the fundamental portfolio optimization problem itself. For example, El Ghaoui et al. (2003) addressed the robust portfolio optimization problem by analysing VaR figures. They assume that only upper and lower bounds to the input parameters are known. Finding the portfolio with the optimized worst-case $\mathrm{VaR}$ is then shown to be an SDP problem.

In an interesting article, Ndaiye et al. (2006) proposed an SDP program with dual convex algorithms for correlation matrices that develops a global risk model. This global risk model is formed by adopting a bottom-up approach which begins with a set of local market covariances as well as capturing the cross correlation between the local markets. This research has shown that SDP holds strong potential for developing robust risk management models for applied users. Later, Lobo et al. (2007) examined ways to optimize a portfolio when linear and fixed transaction

\footnotetext{
${ }^{4}$ The general theory of robustness, which means handling uncertainty, within SDP is discussed by Ben-Tal et al. $(2000,2009)$.
} 
costs are included. The incorporation of transaction costs captures the effect of financial frictions on the optimal allocation of resources. Similarly, Leibfritz and Maruhn (2009) generate a robust portfolio strategy against model errors as a worst-case design. They show how the resulting problem can be solved using SDP sequentially.

The following sub-section presents more recent (post 2010) articles - some are discussed in more detail to highlight recent publications that take a particularly novel approach.

\subsubsection{Post 2010}

Minami (2013) used SDP for portfolio optimization to directly address the issue that the true variance and covariance values can never be observed as they can only be estimates from sample data. Minami used SDP to calculate the maximum value of variance to avoid the sampling error issue.

Chen et al. (2011) study the problem of robust portfolio selection. They demonstrate an efficient procedure for this problem despite the non-robust version being known to be NP-hard in the general case. Sheng-zhi and Fu-sheng (2011) examine higher order moments in portfolio selection and the effectiveness of SDP relaxation via an empirical analysis. In examining a similar problem, Ye et al. (2012) rewrite the robust Markowitz mean-variance portfolio selection problem within the SDP formalism and empirically demonstrate that the resultant portfolios are not as sensitive to input errors as the classic mean-variance portfolios. Using a different approach of applying the Lagragian relaxation to the primal problem, Gao and Li (2013) show that geometric symmetry exists which can be mathematically exploited. In making use of various geometric schemes, they are able to develop efficient polynomial time implementations for the corresponding dual problem. This permits an SDP formulation, using branch-and-bound trimming, which generates high-quality feasible solutions.

By elevating vector variables to positive semidefinite matrix variables, Ling et al. (2014) reformulate robust tracking portfolio models within the SDP framework. They find that compared to the variance tracking error portfolio model, and an equally weighted strategy, their models are more stable and exhibit higher accumulated returns and Sharpe ratios when tested on the SSE50 of the Shanghai Stock Exchange in the majority of observed instances.

Given the known issue with finding the near-optimal solutions for returns on portfolio models in realistic time frames, Gotoh and Fujisawa (2014) propose a time efficient fractional model wherein the ratio of two convex functions is maximized. Their computational results utilized both local and global search algorithms. This approach is similar to earlier work of Yao et al. (2006) which tracked a continuously compounded rate of growth or a stock market index from the dynamic management of a small number of market traded stocks.

Boyd et al. (2014) use SDP to develop a numerical bound on the optimal performance for problems where an optimal policy cannot be determined. The model built captures many features of real multi-period portfolio optimization problems and provides a nearly optimal trading policy along with an upper bound on how suboptimal it can be. The article considers a dynamically traded asset portfolio in a finite time horizon with the goal being to maximize the portfolio returns 
while respecting given constraints. The total expected revenue function not only accounts for realworld factors such as transaction costs and other fees, but it also compares when all the costs were convex-quadratic with the general nonquadratic case. One of the strengths of this work is that the study contains details of an approximate dynamic programming heuristic that determines the trades to be carried out at each discrete step of the finite portfolio time horizon. In particular, the multi-period investment problem is formulated as a stochastic control problem.

Kim et al. (2016) investigate a subset of the general issue of sparse tangent portfolio selection via semi-definite relaxation and propose an approach to resolve the cardinality problem. The problem is that the mean-variance model determines optimal portfolios with high cardinality, thus potentially preventing the model being used in practice because of high transaction costs and management fees. This paper shows an application of the semidefinite relaxation method to a cardinality constrained Sharpe ratio maximization problem. The validity of this method is demonstrated through an empirical test with historical stock returns. Numerical analyses confirm that by solving the relaxed SDP problem, investors are able to effectively construct sparse tangent portfolios. The importance of this is that the resultant portfolios now have reduced cardinality and investors are able to benefit from diversification at a lower cost. By employing an innovative scheme using an adaptive approximation algorithm, Tian et al. (2016) develop a fully positive programming formulation of the cardinality constrained portfolio selection problem. Their work is important because their numerically derived results produce higher quality feasible solutions than other methods discussed in prior literature.

$\mathrm{Xu}$ et al. (2017) use a hybrid genetic algorithm to solve the NP-hard problem with the goal of maximizing the excess return for both passive and active fund management strategies with chance and cardinality constraints. They employ an SDP approximation for the model subject to different sets of potential distribution functions. Their numerical experimentation on real datasets shows that their model and the solution techniques provide good out-of-sample excess returns with a high level of robustness.

Fonseca and Rustem (2012a); Fonseca et al. (2011a, 2012) examined how multi-period international portfolios can be optimized. The development of solutions to these types of applied problems has previously been limited by their exponential complexity. The interaction between local market conditions, foreign exchange rates and multiple-time periods can result in an intractable model. The authors applied an SDP approach which contains the uncertain return in a non-linear (ellipsoidal) set. A further contribution of their papers is to combine portfolio optimization with VaR. This reflects the increased importance of risk in the post Global Financial Crisis (GFC) period.

Fonseca et al. (2011b) examined how SDP can be applied to currency portfolios. The authors examined currency investment strategies where the portfolio is composed only of different currencies. Since currencies are traded as pairs optimal portfolio formation can be more complex. Fonseca et al. used SDP to optimize the allocation of different investments into several different currencies with different patterns of risk and return. The authors also showed how currency options can be laid over the baseline strategy to reduce risk. 
A key component of portfolio modelling is strategies for the reduction of risk. The nonlinear nature of risk provides substantial scope for the application of SDP. Fonseca and Rustem (2012b) applied a robust optimization approach which specifies a minimum value that the portfolio must be worth in the future. This approach is commonly used by endowments, pension funds, insurance companies and government agencies that have known obligations in the future. The problem is formulated as an SDP problem that combines a set of uncertain outcomes with the objective function.

Yang et al. (2014) propose a distributionally robust return-risk optimization model based on risk control of conditional VaR. They show that using an SDP framework the models can be solved in polynomial time.

Using a two stage portfolio optimization formulation, Ling et al. (2017) analyse the stochastic linear optimization problem where the recourse function is risk-averse as opposed to risk-neutral. They show that the first stage is equivalent to an SDP problem, and consolidate the VaR objective function in the second stage demonstrating that the second problem is NP-hard. Their numerical experiments show that their approach can produce solutions of good quality whilst accounting directly for risk.

\subsection{Option Pricing}

The most common option ${ }^{5}$ is a single asset option where there is one underlying asset, such as a defined number of shares in a listed public company (stock option). The price of these options can be found using the famous Black-Scholes option pricing model (Black and Scholes, 1973), which makes the assumptions that there are no-arbitrage opportunities, and that the underlying asset (stock) follows a log-normal distribution (geometric Brownian motion). However, the latter assumption is usually violated, which often biases the price. Consequently, there has been research into pricing models that do not have the underlying log-normal distribution assumption, and it has been shown that bounds on the option prices can be derived from only the no-arbitrage assumption (Lo, 1987). SDP has been used to derive these bounds based on the moments of the underlying asset price ${ }^{6}$. There has also been research into models that assume distributions other than log-normal; Han et al. (2005) list the major models.

Lo (1987) derived a distribution-free upper bound on a single call option given the mean and variance of the underlying asset price. Extending this result, Boyle and Lin (2003) demonstrate that this type of option is of practical use in the finance industry and show that the upper bound can be obtained using SDP given the first two moments of the joint distribution of returns (means and covariance matrix of the underlying asset prices), which they solve using an IP algorithm. Empirical testing of the SDP solution revealed success with many bounds markedly close to the true values.

\footnotetext{
${ }^{5}$ In this paper, the term option specifically refers to European options that can only be exercised at maturity, unlike American options that can be exercised at any time until maturity.

${ }^{6}$ Only call options have been researched, because put options can be easily calculated from call options.
} 
Bertsimas and Popescu (2000, 2002) also extended the research by Lo (1987) and used SDP to develop a distribution-free tight upper bound on a standard single asset option, given the first $n$ moments of the underlying asset price. Bertsimas and Popescu (2002) extended their work to incorporate transaction costs. Gotoh and Konno (2002) then further extended the work of Bertsimas and Popescu, again using SDP to establish a tight lower bound for a single asset option. They showed that these upper and lower bounds are extremely close to the actual option price, especially as the number of moments becomes large $(n \geq 4)$. In addition, Gotoh and Konno (2002) presented a tailored algorithm to calculate the bounds of a single asset option in real-time ${ }^{7}$, for $n \leq 4$, which includes the largest meaningful problem in practice.

Han et al. (2005) generalized the previous research also using SDP to find the upper bound of multi-asset or basket options (as done in part by Boyle and Lin) by using the first $n$ moments of the underlying assets (as done by Bertsimas and Popescu, and Gottoh and Konno). As was the case with the previous research, empirical results were positive, but only a small data set was used and consequently more thorough empirical testing would be beneficial. Independently, d'Aspremont (2002a, 2003) also conducted research into basket options, specifically swaptions, which are an option in the interest rate market. He determined that swaptions could be priced as basket options. In addition, for this specific case it was shown that the basket option representing the swaption had underlying assets that were log-normally distributed. Hence, the Black-Scholes option pricing model assumptions were not violated. Consequently, d'Aspremont extended the single asset Black-Scholes model to cater for basket options and found that the resulting problem could be solved efficiently using standard SDP algorithms.

Using SDP, Lasserre et al. (2006) examined an array of exotic options including fixed strike, arithmetic average Asian and down-and-out barrier call options. Their pricing method was to identify derivative prices with an infinite-dimensional linear programming problem involving the moments. By then applying SDP, the problem is transformed into a finite dimension problem. Numerical results were found with a small number of sample moments.

Li and Kwon (2012) applied SDP techniques to derivative problems. They examined convex risk measures based on the market prices of derivative instruments, motivated by the work of Lo (1987) who considered a semi-parametric approach. The authors incorporate infinite families of distributions to provide a distribution free-optimization approach. Li and Kwon were able to show theoretically and empirically that reformulating the measurement of options' risk measures as SDP problems which can be solved efficiently and be regarded as distribution free. This is an important advancement as a limitation of several well-known finance theories in that they require distributional assumptions that do not hold empirically.

In his second $\mathrm{PhD}$ thesis, d'Aspremont (2002b) addressed the problem of determining the presence of arbitrage opportunities with basket options (such as swaptions). Specifically, the following problem was addressed: 'Given a set of basket options, what are the possibilities for arbitrage?'. Bertsimas and Popescu (2002) had previously shown that this problem was NP-hard,

\footnotetext{
7 The bounds were calculated in less than 0.1 seconds on a now old computer (Pentium III 500MHz).
} 
and consequently that there is no efficient method for finding all arbitrage opportunities. Therefore, d'Aspremont focused on detecting economically significant arbitrage opportunities with low computational cost by calculating price bounds for the basket options based on other related basket options. However, the following unrealistic assumptions were made: no transaction costs, and arbitrage opportunities were limited to trading on the day of analysis and at maturity. Nevertheless, increasingly tight bounds were able to be obtained by solving progressively larger and more complex SDP problems. This technique was not tested on real market data. Zymler et al. (2013) explicitly captured the nonlinear risk of a portfolio of derivatives by applying a worst case $\mathrm{VaR}$ approach. They developed two conservative approximations for VaR and empirically demonstrated via numerical experiments the benefits of incorporating SDP to capture the nonlinear risk of a derivative portfolio. A key advantage of this semidefinite approach to risk management is that it does not require knowledge of the underlying option pricing models.

Cuturi and D'aspremont (2013) present several techniques for examining paths of multivariate stochastic processes and show that many of these optimization processes can be solved exactly using SDP. They discuss how these methods can be used to isolate statistical arbitrage opportunities.

In a more recent article, the problem of computing bounds on option pricing under regime switching is tackled by Kwon and Li (2016). This problem arises from the desire to find an alternative to the standard geometric Brownian motion in the Black-Scholes framework as it often results in pricing biases. Prior research conducted by Bertsimas and Popescu $(2000,2002)$ had shown that the problem of finding the tightest bounds can be cast as an SDP problem. This study extends on this result by incorporating a regime-switching model for the underlying asset in computing bounds for European-style call options, where the regime-switching framework is able to capture changing volatility in asset returns. This research is important as it compares and contrasts SDP to classic finance techniques. Kwon and Li show that by incorporating a finite-state Markov chain regime-switching process, a discrete lattice is generated for computing the option bounds. The two-stage simple stochastic SDP with a recourse model aims to find a current (firststage) bound for an option price via the semidefinite model of Bertsimas and Popescu as well as obtaining second-stage option bounds through SDP. The optimal solution is then found through sorting the option pricing bounds. The stochastic SDP models developed allow the computation of a bounded price for European call options. The striking feature of the modelling is that by using a stochastic programming technique, the bounds can be calculated in a straightforward manner where those bounds are controlled through penalty parameters that explicitly include risk aversion. The empirical performance of the calculated robust bounds is investigated through rather extensive numerical experiments using S\&P500 stock index options. The results suggest such a stochastic approach is more flexible when determining the pricing bounds, as opposed to the use of deterministic models. This is also an effective approach in that the models are tractable and controllable through the penalty parameters. 


\subsection{Future Research Directions}

This section considers future research opportunities within the existing streams of portfolio optimization and option pricing. Opportunities to apply SDP to new areas are also presented, including worst-case optimizations in finance contexts and environmental finance. Other areas of future research include risk management, asset and liability management and market equilibrium analysis.

\subsubsection{Existing Streams of Portfolio Optimization and Option Pricing}

Researchers have recently been investigating portfolio optimization under three criteria (see e.g. Qi et al., 2017), instead of the standard two criteria of mean and variance. For example, using skewness as the third criterion, Briec et al. (2013) develop a polynomial-based solution to the problem of maximizing the return and skewness optimization. The authors assumed unit variance, but point out that this is always achievable through rescaling. SDP could be used to add robustness and scalability to these problems.

The development and use of SDP within the options research space is more embryonic. Han et al. (2005) demonstrated the power of SDP in robustly handling large datasets. An area of further research interest would be comparing SDP with other approaches. For example, the efficiency of Alcock and Smith's (2017) unique non-parametric approach to value American options using Cressie-Read divergences, could be compared to an SDP solution.

\subsubsection{Worst-Case Optimization}

Problems involving worst-case linear optimization (WCLO) under uncertainties abound in finance. In a strongly mathematical analysis, Peng and Zhu (2015) show that worst-case linear optimization (WCLO) relaxation applications, with uncertainties in their constraints, belong to the NP-hard problem set. By presenting a coarse semidefinite relaxation for WCLO, the study details a numerical technique involving an iterative procedure to sequentially refine and tighten the relaxation model. It is then demonstrated that this sequence will converge to a non-linear SDP problem. This resulting problem is resolved through a bi-section search algorithm, and global convergence of the algorithm is established. The authors show that their preliminary experimental results demonstrate that the proposed algorithm finds very tight bounds for the original WCLO and is also able to locate an exact global optimal solution for most tested instances. This result is important as the method developed is applicable to many optimization problems in finance including applications in systemic risk estimates and stochastic optimization.

\subsubsection{Environmental Finance}

Environmental finance has recently been identified as an important, emerging, interdisciplinary field in Asia-Pacific finance (Linnenluecke, 2016, 2017). Linnenluecke et al. (2017) encouraged researchers to be more open to use new methodologies. SDP is a technique that is well suited to contribute to research in this field, particularly considering the additional 
nonlinear environmental constraints that do not occur in non-traditional portfolio optimization and real option valuation problems.

Using SDP Costa et al. (2017) find a solution to an electricity energy mix that reconciles $\mathrm{CO} 2$ emission, risk and associated costs under uncertainty for the electricity portfolio optimization problem that exists in Brazil. In showing this, they are able to use their approach to solve three separate energy portfolio problem formulations successfully. Investigating the same fundamental problem set in the European Union, Eckhause and Herold (2014) employ a real options framework to determine optimal project selection and funding solutions to achieve an operating full-scale CO2 capture, transport and storage plant by a target year of 2020 . They achieve this by casting and solving a model using SDP and demonstrate that they achieve an improved risk reduction considering sensitivity to budget flexibility whilst optimizing overall budget expenditure.

It would be interesting to contrast the results above with Emissions Trading Schemes (ETSs) developed for Australia and NZ (see, for example, Bui et al. (2017) and Chapple et al. (2013) for an analysis of these ETSs). Furthermore, it would be interesting to extend the comparisons to include non-SDP solutions.

A yet unexplored area is to examine the applications of SDP in the area of real options for natural resource investments - an area pioneered by the seminal paper of Brennan and Schwartz (1985). The limiting factor is the number of assets that can be considered using extensions of Brennan and Schwartz's approach; currently finding a solution typically involves simulation or solution techniques such as finite differences (see Kelly (2017) for a recent example). SDP allows problems with many assets to be solved more efficiently than other techniques. This is of particular interest given the widespread interest in renewable, sustainable assets. Linnenluecke et al. (2017) explain that renewables represent valuation challenges and that new methods along the lines of real options would prove valuable.

\subsubsection{Other Areas}

Risk Management: option pricing is NP-Hard. d'Aspremont (2002a) has extended prior work in option pricing to develop hedging strategies using options for risk management. Yamada and Primbs (2002) have also shown that option hedging strategies can be represented in part by moment problems, which have been solved successfully using SDP.

Asset and Liability Management: very difficult optimization problems related to maximizing the growth of a company whilst managing its risks and conforming to regulatory requirements exist in this area. A common example of an industry affected by this is banking. Banks are affected across several dimensions, including interest rates, liquidity and solvency. Linear programming has been used on simplified problems with few constraints suggesting that SDP could be used to address more complex problems with more (non-linear) constraints.

Market Equilibrium Analysis: finding the conditions for which the market is in equilibrium. SDP would be useful within this application domain as special cases of market equilibrium are convex optimization problems. Furthermore, many market equilibrium problems can be solved by 
similar techniques to that of portfolio optimization (Nagurney, 2002), which is a major financial application of SDP.

\section{Conclusion}

This review has demonstrated the benefits of using SDP in a finance context is that it is more robust than MMV modelling, is able to handle more complex, non-linear constraints and deal with portfolios with a larger number of assets. For example, the Markowitz Mean-Variance model (MMV) is often criticized in practice because it is highly sensitive to the mean return of the underlying assets, but SDP provides more robust estimates and can also incorporate higher moments such as skewness and kurtosis.

Overall, SDP has been successfully applied to many financial optimization problems with particular success in forming optimal robust portfolios including multi-period strategies and pricing options without restrictive distributional assumptions. Using SDP, it would be possible in these areas to use larger data sets.

SDP is a standard optimization technique, but its use in finance appears to have been restricted by a lack of introductory papers and publications in finance journals. This paper aims to bridge this gap by providing an overview of the SDP literature and drawing attention to existing financial applications of SDP with a view to encouraging interdisciplinary research in this area.

A solid platform of SDP theory has been established and efficient algorithms exist to solve SDP problems. Research focus is now needed into the applications of SDP, which can often be identified as those found too complex to be represented as linear programs. Applications in finance such as portfolio optimization and option pricing are a prime example. Opportunity also exist for future research in worst-case optimization and the emerging area of environmental finance, particularly SDP approaches to optimization of energy assets and real option valuation approaches for renewable resources.

In other areas of finance that have traditionally relied on linear programming for solutions, SDP could potentially play a role where the true constraints are non-linear. An example of this is in capital budgeting when there is uncertainty associated with input parameters, which means they are stochastic rather than constant, and traditional linear programming techniques are unsuitable. SDP could also play a role in developing models that are free from traditional assumptions of the distribution of asset returns. 


\section{References}

Alcock, J. and G. Smith, 2017, Non-parametric American option valuation using Cressie-Read divergences, Australian Journal of Management 42(2), 252-275.

Alizadeh, F., 1991, Combinatorial optimization with interior point methods and semidefinite matrices, Ph.D. thesis (University of Minnesota).

Alizadeh, F., 1992, Optimization over the positive-definite cone: Interior point methods and combinatorial applications, in: Pardalos, P. M., ed., Advances in Optimization and Parallel Computing, 1-25.

Anton, H., and C. Rorres, 2000, Elementary Linear Algebra: Applications Version, eighth ed (John Wiley and Sons, New York).

Audenaert, K., and B. De Moor, 2002, Optimizing completely positive maps using semidefinite programming, Physical Review A 65 (030302), 1-4.

Balakrishnan, V., and F. Wang, 2000, SDP in Systems and Control Theory, in: Wolkovitz (2000), 421-441.

Bellman, R., and K. Fan, 1963, On systems of linear inequalities in hemitian matrix variables, in: Klee, V. L., ed., Convexity, Proceedings of the Symposia in Pure Mathematics, Vol. 7 (American Mathematics Society), 1-13.

Ben-Tal, Aharon, Laurent El Ghaoui, and Arkadi Nemirovski. 2000. Robustness. In Wolkovitz (2000), 139-162.

Ben-Tal, A., L. El Ghaoui, and A. Nemirovskii, 2009, Robust optimization (Princeton University Press, Princeton, NJ).

Benson, S. J., 2003, Parallel Computing on Semidefinite Programs, Technical report ANL/MCSP939-0302 (Mathematics and Computer Science Division, Argonne National Laboratory).

Benson, S. J., Y. Ye, and X. Zhang, 2000, Solving Large-Scale Sparse Semidefinite Programs for Combinatorial Optimization. SIAM Journal on Optimization 10 (2): 443-461.

Bertsimas, D., and I. Popescu, 2000, Moment Problems via Semidefinite Programming: Applications in Probability and Finance, Technical report 2000/27/TM (INSEAD, Fontainebleau, France).

Bertsimas, D., and I. Popescu, 2002, On the Relation between Option and Stock Prices: A convex optimization approach, Operations Research 50 (2), 358-374.

Black, F., and M. Scholes, 1973, The pricing of options and corporate liabilities, Journal of Political Economy 81, 637-654.

Borchers, B., 1999a, CSDP 2.3 user's guide, Optimization Methods and Software 11(1-4), 597611.

Borchers, B., 1999b, CSDP: A C library for semidefinite programming, Optimization Methods and Software 11(1-4), 613-623.

Borchers, B., and J. G. Young, 2007, Implementation of a primal-dual method for SDP on a shared memory parallel architecture, Computational Optimization and Applications 37(3), 355-369.

Borwein, J. M., and H. Wolkowicz, 1980, Characterization of optimality for the abstract convex 
program with finite-dimensional range, Journal of the Australian Mathematical Society Series A 30(4), 390-411.

Boyd, S., L. El Ghaoui, E. Feron, and V. Balakrishnan, 1994, Linear Matrix Inequalities in System and Control Theory (Volume 15 of SIAM Studies in Applied Mathematics, Society for Industrial and Applied Mathematics (SIAM), Philadelphia).

Boyd, S., M. T. Mueller, B. O’Donoghue, Y. Wang, 2014, Performance Bounds and Suboptimal Policies for Multi-Period Investment, Foundations and Trends in Optimization 1(1), 1-72.

Boyle, P., and X. S. Lin, 2003, Bounds on Multiple Contingent Claims Based on Several Assets, Journal of Financial Economics 46, 383-400.

Bravo, H., 2016, R Interface to the CSDP Semidefinite Programming Library. Available at: $C R A N$ Repository https://cran.r-project.org/web/packages/Rcsdp/Rcsdp.pdf.

Brennan, M. J., and E. S. Schwartz, 1985, Evaluating natural resource investments, Journal of business 58(2), 135-157.

Briec, W., K. Kerstens, and I. Van de Woestyne, 2013, Portfolio selection with skewness: A comparison of methods and a generalized one fund result, European Journal of Operational Research 230(2), 412-421.

Bui, B., Chapple, L., \& Truong, T. P. (2017). Drivers of tight carbon control in the context of climate change regulation. Accounting \& Finance.

Chapple, L., P.M. Clarkson, and D.L. Gold, 2013, The cost of carbon: Capital market effects of the proposed emission trading scheme (ETS), Abacus 49(1), 1-33.

Chen, L., S. He, and S. Zhang, 2011, Tight bounds for some risk measures with applications to robust portfolio selection, Operations Research 59(4), 847-865.

Costa, O.L.V., C. de Oliveira Ribeiro, E. E. Rego, J. M. Stern, V. Parente, and S. Kileber, 2017, Robust portfolio optimization for electricity planning: An application based on the Brazilian electricity mix, Energy Economics 64, 158-169.

Costa, O.L.V., and A. C. Paiva, 2002, Robust Portfolio Selection Using Linear Matrix Inequalities, Journal of Economic Dynamics and Control 26, 889-909.

Cuturi, M., and A. D'aspremont, 2013, Mean reversion with a variance threshold, in: Proceedings of the 30th International Conference on Machine Learning (ICML-13), 271-279.

Cvetović, D., M. Cangalović, and V. Kovačevič-Vujcić, 1999, Semidefinite Programming Methods for the Traveling Salesman Problem, in: Proceedings of the 7th International Integer Programming and Combinatorial Optimization (IPCO) Conference, 126-136.

Dantzig, G. B., 1997, Linear Programming (Springer Series in Operations Research, Springer, New York).

d'Aspremont, A. W., 2002a, Interest rate model calibration and risk-management using semidefinite programming, Ph.D. thesis (Ećole Polytechnique).

d'Aspremont, A. W., 2002b, Shape Constrained Optimization, with Applications in Finance and Engineering. Ph.D. thesis (Stanford University).

d'Aspremont, A. W., 2003, Interest Rate Model Calibration Using Semidefinite Programming, Applied Mathematical Finance 10(3), 183-213. 
de Klerk, E., 2002, Aspects of Semidefinite Programming: Interior Point Algorithms and Selected Application (Kluwer Academic Publishers, Dordrecht, The Netherlands).

de Klerk, E., D. Pasechnik, and J. Warners, 2004, On approximate graph colouring and MAX-kCUT algorithms based on the $\theta$-function, Journal of Combinatorial Optimization 8, 267294.

Eckhause, J., and J. Herold, 2014, Using real options to determine optimal funding strategies for CO 2 capture, transport and storage projects in the European Union, Energy Policy 66, 115134.

El Ghaoui, L., M. Oks, and F. Oustry, 2003, Worst-Case Value-At-Risk and Robust Portfolio Optimization: A Conic Programming Approach, Operations Research 51(4), 543-556.

Fedorov, V., 2000, Design of Regression Experiments, in: Wolkovitz (2000), 511-528.

Fonseca, R.J., W. Wiesemann, and B. Rustem, 2011, A Semidefinite Programming Approach to Portfolio Optimization, Computer Aided Chemical Engineering 29, 472-476.

Fonseca, R.J., W. Wiesemann, and B. Rustem, 2012, Robust international portfolio management, Computational Management Science 9(1), 31-62.

Fonseca, R. J., and B. Rustem, 2012a, International portfolio management with affine policies, European Journal of Operational Research 223(1), 177-187.

Fonseca, R. J., and B. Rustem, 2012b, Robust hedging strategies, Computers and Operations Research 39(11), 2528-2536.

Fonseca, R. J., S. Zymler, W. Wiesemann, and B. Rustem, 2011, Robust optimization of currency portfolios, Journal of Computational Finance 15(1), 3-30.

Freund, R. M., 2004, Introduction to Semidefinite Programming (SDP), Technical report (Massachusetts Institute of Technology).

Frieze, A. M., and M. Jerrum, 1995, Improved Approximation Algorithms for MAX k-CUT and MAX BISECTION, in: Proceedings of the 4th International Integer Programming and Combinatorial Optimization (IPCO) Conference (Springer-Verlag, London), 1-13.

Fujisawa, K, M. Kojima, and K. Nakata, 1997, Exploiting sparsity in primal-dual interior-point methods for semidefinite programming, Mathematical programming 79(1-3), 235-253.

Gao, J., and D. Li, 2013, Optimal cardinality constrained portfolio selection, Operations research 61(3), 745-761.

Gärtner, B., and J. Matoŭsek, 2012, Approximation Algorithms and Semidefinite Programming (Springer, Berlin).

Goemans, M., and F. Rendl, 2000, Combinatorial Optimization, in: Wolkovitz (2000), 343-360.

Goemans, M., and D. P. Williamson, 1995, Improved approximation algorithms for maximum cut and satisfiability problems using semidefinite programming, Journal of the Association of Computing Machinery 42, 1115-1145.

Goldfarb, D., and G. Iyengar, 2003, Robust portfolio selection problems, Mathematics of Operations Research 28(1), 1-38.

Gotoh, J., and H. Konno, 2002, Bounding option prices by semidefinite programming: a cutting plane algorithm, Management Science 48(5), 665-678. 
Gotoh, J., and K. Fujisawa, 2014, Convex optimization approaches to maximally predictable portfolio selection, Optimization 63(11), 1713-1735.

Grötschel, M., L. Lovász, and A. Schrijver, 1988. Geometric Algorithms and Combinatorial Optimization (Springer-Verlag, Berlin, New York).

Hadley, G., 1969, Linear Programming (World Student Series, Addison-Wesley Publishing Company).

Han, D., X. Li, D. Sun, and J. Sun, 2005, Bounding option prices of multi-assets: a semidefinite programming approach, Pacific Journal of Optimization 1(1), 59-79.

Hatherley, A. and J. Alcock, 2007, Portfolio construction incorporating asymmetric dependence structures: a user's guide, Accounting \& Finance 47(3), 447-472.

Helmberg, C., 2000, Semidefinite Programming for Combinatorial Optimisation (Konrad-ZuseZentrum für Informationstechnik, Berlin-Dahlem, Germany).

Helmberg, C., and F. Outsry, 2000, Bundle Methods and Eigenvalue Functions, in: Wolkovitz (2000), 307-337.

Helmberg, C., and R. Weismantel, 1998, Cutting Plane Algorithms for Semidefinite Relaxations, in: Pardalos and Wolkowicz (1998b), 197-213.

Horn, R. A., and C. R. Johnson, 1985, Matrix Analysis (Cambridge University Press).

Horn, R. A., and C. R. Johnson, 1991, Topics in Matrix Analysis (Cambridge University Press).

Kamath, A., and N. K. Karmarkar, 1992, A continuous method to compute upper bounds in quadratic maximization problems with integer constraints, in: Floudas, C. A., and P. M. Pardalos, eds., Recent Advances in Global Optimization (Princeton University Press, Princeton, New Jersey), 125-140.

Kamath, A., and N. K. Karmarkar, 1993, An O(nL) iteration algorithm for computing bounds in quadratic optimization problems, in: Pardalos, P.M., ed., Complexity in Numerical Optimization (World Scientific Publishing Company, Singapore), 254-268.

Karger, D., R. Motwani, and M. Sudan, 1998, Approximate Graph Coloring by Semidefinite Programming, Journal of the Association of Computing Machinery 45(2), 246-265.

Karmarkar, N. K., 1984, A new polynomial-time algorithm for linear programming, Combinatorica 4, 373-395.

Kelly, S., 2017, The market premium for the option to close: evidence from Australian gold mining firms, Accounting \& Finance 57, 511-531.

Kim, M.J., Y. Lee, J.H. Kim, and W.C. Kim, 2016, Sparse tangent portfolio selection via semidefinite relaxation, Operations Research Letters 44(4), 540-543.

Koenker, R., and I. Mizera, 2014, Convex Optimization in R, Journal of Statistical Software $60(5), 1-23$.

Kwon, R.H., and J.Y. Li, 2016, A stochastic semidefinite programming approach for bounds on option pricing under regime switching, Annals of Operations Research 237(1-2), 41-75.

Lasserre, J. B., T. Prieto-Rumeau, and M. Zervos, 2006, Pricing a class of exotic options via moments and SDP relaxations, Mathematical Finance 16(3), 469-494.

Laurent, M., 1998, A Tour d'Horizon on Positive Semidefinite and Euclidean Distance Matrix 
Completion Problems, in: Pardalos and Wolkowicz (1998b), 51-76.

Laurent, M., and F. Rendl, 2002, Semidefinite Programming and Integer Programming, Technical report PNA-R0210 (CWI, Amsterdam).

Lee, J., 2000, Semidefinite programming in experimental design, in: Wolkovitz (2000), 528-532.

Leibfritz, F., and J. H. Maruhn, 2009, A successive SDP-NSDP approach to a robust optimization problem in finance, Computational Optimization and Applications 44(3), 443-466.

Lewis, A. S., and M. L. Overton, 1996, Eigenvalue Optimization, Acta Numerica 5, 149-190.

Li, J. Y., and R. H. Kwon, 2012, Market price-based convex risk measures: A distribution-free optimization approach, Operations Research Letters 40(2), 128-133.

Li, S., Y. Zhou, Y. Wu, and X. Ge, 2017, Equilibrium approach of asset and option pricing under Lévy process and stochastic volatility, Australian Journal of Management 42(2), 276-295.

Ling, A., J. Sun, N. Xiu, and X. Yang, 2017, Robust two-stage stochastic linear optimization with risk aversion, European Journal of Operational Research 256(1), 215-229.

Ling, A., J. Sun, and X. Yang, 2014, Robust tracking error portfolio selection with worst-case downside risk measures, Journal of Economic Dynamics and Control 39, 178-207.

Linnenluecke, M.K., X. Chen, X. Ling, T. Smith, and Y. Zhu, 2016. Emerging trends in AsiaPacific finance research: A review of recent influential publications and a research agenda, Pacific-Basin Finance Journal 36, 66-76.

Linnenluecke, M.K., X. Chen, X. Ling, T. Smith, and Y. Zhu, 2017, Research in finance: A review of influential publications and a research agenda, Pacific-Basin Finance Journal 43, $188-199$.

Lo, A. W., 1987, Semi-parametric upper bounds for options prices and expected payoffs, Journal of Financial Economics 19, 373-387.

Lobo, M. S., M. Fazel, and S. Boyd, 2007, Portfolio optimization with linear and fixed transaction costs, Annals of Operations Research 152(1), 341-365.

Lovász, L., 1979, On the Shannon capacity of a graph, IEEE Transactions on Information Theory $25,1-7$.

Low, R. K. Y. (2018). Vine copulas: modelling systemic risk and enhancing higher-moment portfolio optimisation. Accounting \& Finance, 58, 423-463.

Lutgens, F., and J. Sturm, 2002, Robust one period option modelling, Technical report 114

(Center for Economic Research, Tilburg University, Netherlands).

Markowitz, H., 1952, Portfolio Selection, Journal of Finance 7(1), 77-91.

Markowitz, H., 1959, Portfolio Selection: Efficient Diversification of Investments (John Wiley, New York).

Minami, S., 2013, Robust Portfolio Optimization under Sampling Error, SSRN Working Paper.

Mirkin, B., 1998, Approximation Clustering: a Mine of Semidefinite Programming Problems, in: Pardalos and Wolkowicz (1998b), 167-180.

Mishra, A.V., 2016, Foreign bias in Australian-domiciled mutual fund holdings, Pacific-Basin Finance Journal 39, 101-123.

Nagurney, A., 2002, Optimization in the Financial Services Industry, in: Pardalos and Resende 
(2002), 901-917.

Ndaiye, P. M., F. Oustry, and V. Piolle, 2006, Semidefinite optimisation for global risk modelling, Journal of Asset Management 7, 142-153.

Nesterov, Y. E., and A. S. Nemirovskii, 1988, A general approach to polynomial-time algorithms design for convex programming, Technical report (Central Economic \& Mathematical Institute, USSR Academy of Sciences, Moscow).

Nesterov, Y. E., and A. S. Nemirovskii, 1990a, Optimization over positive semidefinite matrices:

Mathematical background and user's manual, Technical report (Central Economic \& Mathematical Institute, USSR Academy of Sciences, Moscow).

Nesterov, Y. E., and A. S. Nemirovskii, 1990b, Self-concordant functions and polynomial time methods in convex programming, Technical report (Central Economic \& Mathematical Institute, USSR Academy of Sciences, Moscow).

Nesterov, Y. E., and A. S. Nemirovskii, 1994, Interior-Point Polynormial Algorithms in Convex Programming (Studies in Applied Mathematics (SIAM), Philadelphia).

O’Donoghue, B., E. Chu, N. Parikh, and S. Boyd, 2016a, Conic Optimization via Operator Splitting and Homogeneous Self-Dual Embedding, Journal of Optimization Theory and Applications 169(3): 1042-1068.

O’Donoghue, B., E. Chu, N. Parikh, and S. Boyd, 2016b, SCS: Splitting Conic Solver, version 1.2.7. Available at https://github.com/cvxgrp/scs.

Qi, Y., R.E. Steuer, and M. Wimmer, 2017, An analytical derivation of the efficient surface in portfolio selection with three criteria, Annals of Operations Research 251(1-2), 161-177.

Pardalos, P., and H. Wolkowicz, 1998a, Preface, in: Pardalos and Wolkowicz (1998b), vii-xx. Pardalos, P. M., and H. Wolkowicz, eds., 1998b, Topics in Semidefinite and Interior-Point Methods (Volume 18 of The Fields Institute for Research in Mathematical Sciences, Communication Series, American Mathematical Society, Providence, Rhode Island).

Pardalos, P. M., and M. G. C. Resende, eds., 2002, Handbook of Applied Optimization (Oxford University Press, New York).

Peng, J., and T. Zhu., 2015, A nonlinear semidefinite optimization relaxation for the worst-case linear optimization under uncertainties, Mathematical Programming 152(1-2), 593-614.

Porkolab, L., and L. Khachiyan, 1997, On the complexiy of semidefinite programs, Journal of Global Optimisation 10(4), 351-365.

Porkolab, L., and L. Khachiyan, 1998, Testing the feasibility of Semidefinite Programs, in: Pardalos and Wolkowicz (1998b), 17-26.

Ramana, M. V., 1997, An exact duality theory for semidefinite programming and its complexity implications, Mathematical Programming 77, 129-162.

Ramana, M. V., Levent Tunçel, and H. Wolkowicz, 1997, Strong Duality for Semidefinite Programming, SIAM Journal on Optimization 7(3), 641-662.

Roll, R., 1992, A mean/variance analysis of tracking error, The Journal of Portfolio Management $18,13-22$.

Sheng-zhi, P., and W. Fu-sheng, 2011, Semidefinite programming relaxation for portfolio 
selection with higher order moments, in: 2011 International Conference on Management Science Engineering 18th Annual Conference Proceedings, 99-104.

Skutella, M., 2001, Convex quadratic and semidefinite programming relaxations in scheduling, Journal of the Association of Computing Machinery (ACM) 48, 206-242.

Smith, T. and K. Walsh, 2013, Why the CAPM is Half-Right and Everything Else is Wrong, Abacus 49(S1), 73-78.

Suh, S., 2016, A Combination Rule for Portfolio Selection with Transaction Costs, International Review of Finance 16(3), 393-420.

Tian, Y., S. Fang, Z. Deng, and Q. Jin, 2016, Cardinality Constrained Portfolio Selection Problem: A Completely Positive Programming Approach, Journal of Industrial and Management Optimization, 12(3), 1041-1056.

Vandenberghe, L., and S. Boyd, 1996, Semidefinite Programming, SIAM Review 38, 49-95. Wolkowicz, H., ed., 2000, Handbook of Semidefinite Programming: Theory, Algorithms, and Applications (Kluwer Academic Publishers, Boston).

Wolkowicz, H., 2002, Semidefinite Programming, in: Pardalos and Resende (2002), 40-50.

Wolkowicz, H., and M. F. Anjos, 2002, Semidefinite programming for discrete optimization and matrix completion problems, Discrete Applied Mathematics 123(1-3), 513-577.

Wolkowicz, H., R. Saigal, and L. Vandenberghe, 2000, Introduction, in Wolkowicz (2000), 1-8.

Wright, S. J., 2004, Nonlinear and Semidefinite Programming, in Proceedings of Symposia in Applied Mathematics, Vol. 61 (American Mathematical Society), 115-137.

Xu, F., M. Wang, Y. Dai, and D. Xu, 2017, A sparse enhanced indexation model with chance and cardinality constraints, Journal of Global Optimization, 1-21.

Yamada, Y., and J. A. Primbs, 2002, Value-at-Risk estimation for dynamic hedging, International Journal of Theoretical and Applied Finance 5(4), 333-354.

Yamashita, M., K. Fujisawa, M. Fukuda, K. Kobayashi, K. Nakata, and M. Nakata, 2012, Latest developments in the SDPA family for solving large-scale SDPs, in: Handbook on semidefinite, conic and polynomial optimization (Springer), 687-713.

Yamashita, M., K. Fujisawa, and M. Kojima, 2003, SDPARA: SemiDefinite Programming Algorithm paRAllel version, Journal of Parallel Computing 29(8), 1053-1067.

Yang, L., Y. Li, Z. Zhou, and K. Chen, 2014, Distributionally robust return-risk optimization models and their applications, Journal of Applied Mathematics 2014: 1-9.

Yao, D., S. Zhang, and X. Y. Zhou, 2006, Tracking a financial benchmark using a few assets, Operations Research 54(2), 232-246.

Ye, Kai, P. Parpas, and B. Rustem, 2012, Robust portfolio optimization: a conic programming approach, Computational Optimization and Applications 52(2), 463-481.

Zhu, Z., and Y. Ye, 2016, R Interface to DSDP Semidefinite Programming Library. Avaialble at CRAN Repository, https://cran.r-project.org/web/packages/Rdsdp/Rdsdp.pdf.

Zymler, S., D. Kuhn, and B. Rustem, 2013, Worst-Case Value at Risk of Nonlinear Portfolios, Management Science 59(1), 172-188. 


\section{Appendix A - Explanation of some background concepts}

For further background material, Horn and Johnson $(1985,1991)$ contain excellent treatments of matrix analysis and matrix algebra and Anton and Rorres (2000) have written an excellent textbook on linear algebra with many example problems. A more concise review of linear algebra pertinent to SDP is given by Helmberg (2000, ch. 1).

\section{A.1 Inner Product of Symmetric Matrices}

Similar to the inner product of vectors, the inner product of symmetric matrices is a way to multiply two symmetric matrices $(C, X)$ to produce a scalar. In fact, the inner product of $C$ and $X$, denoted $C \bullet X$ or $\langle C \mid X\rangle$, is a linear function that equals

$$
\sum_{i=1}^{m} \sum_{j=1}^{n} C_{i, j} \times X_{i, j}
$$

where $C_{i, j}$ (or $X_{i, j}$ ) is the element in matrix $C$ (or $X$ ) at row $i$ and column $j . C \bullet X$ is also equivalent to the trace (sum of diagonal elements) of the matrix product of $C$ and $X$, denoted $\operatorname{Tr}(C X)$

\section{A.2 Positive Semidefinite and Positive Definite Matrices}

The first requirement of a positive semidefinite matrix is that it is symmetric. For the symmetric matrix $X$ to also be positive semidefinite, all the eigenvalues of $X$ must be nonnegative real numbers. That is, $k \geq 0$ for all solutions of $X v=k v$, where $k$ is a scalar and $v$ is a $n \times 1$ dimensional vector in $\mathbb{R}^{n}$.

There are also alternative ways of defining a positive semidefinite matrix. The other two common definitions for a positive semidefinite matrix are a symmetric matrix $(X)$ for which:

- $\quad v^{T} X v \geq 0$ for any $n \times 1$ dimensional vector $v$ in $\mathbb{R}^{n}$. Note that $v^{T}$ denotes the transpose of the vector $v$, and is the convention used to denote transpose in this paper.

- The determinants of all the principal submatrices are nonnegative. The principal submatrices of $X$ are formed from the first $r$ rows and $r$ columns of $X$ for $r=1,2, \ldots, n$.

Positive semidefinite constraints (also referred to as linear matrix inequalities (LMI)) are represented as $X \geqslant 0$, which means that the matrix $X$ is positive semidefinite. Furthermore, a common expression in the SDP field is $A \geqslant B$ that means $A-B$ is a positive semidefinite matrix.

A positive definite matrix $(Y)$ is a symmetric matrix with the following equivalent conditions:

- $\quad$ All the eigenvalues of $Y$ are positive,

- $\quad v^{T} Y v>0$ for any $n \times 1$ dimensional vector $v$ in $\mathbb{R}^{n}$, and

- The determinants of all the principal submatrices are positive.

Hence, positive definite constraints are represented as $Y>0$, which means that the matrix $Y$ is positive definite. Note that a positive definite matrix is also a positive semidefinite matrix. 


\section{A.3 Closed Convex Cone}

$K$ is a closed convex cone if both

- $\quad K$ is a convex cone, which means that taking any two points $w, x \in K$ then $\alpha w+\beta x \in K$ for all nonnegative scalars $\alpha$ and $\beta$;

- $\quad K$ is a closed set (in a topological sense), in that $K$ includes all its boundary points.

Closed convex cones are of interest as the set of positive semidefinite matrices, denoted $S_{+}^{n}$, is a closed convex cone. This is demonstrated by taking $W, X \in S_{+}^{n}$ and two nonnegative scalars $\alpha$ and $\beta$, where, for any $v \in \mathbb{R}^{n}$

$$
v^{T}(\alpha W+\beta X) v=v^{T} W v+v^{T} X v \geq 0 .
$$

The above expression is $\geq 0$ as $v^{T} W v$ and $v^{T} X v$ are both $\geq 0$ by definition because $W$ and $X$ are semidefinite matrices. This has shown that $\alpha W+\beta X \in S_{+}^{n}$, and consequently that $S_{+}^{n}$ is a convex cone. In addition, $S_{+}^{n}$ is also closed as it includes its boundary points, indicted by the greater than or equal to nature of its definition. On the other hand, the set of positive definite matrices, denoted $S_{++}^{n}$, is also a convex cone, but is not closed.

\section{A.4 (Basic) Feasible Solutions}

For a given problem, a point in the constraint set (that is, satisfies the constraints) is a feasible solution and the collection of all these points is the feasible region or feasible set. The vertices of a convex ${ }^{8}$ feasible region without any curves are termed basic feasible solutions (BFS), and one of the BFS will satisfy the optimality condition. For convex feasible regions that include curves, the BFS is comprised of the number of vertices and the infinite number of points along all curves.

\section{A.5 Simplex Algorithm}

The Simplex algorithm is a common algorithm used to solve an optimization problem with a polytope feasible region, such as a linear programming (LP) problem. The algorithm has exponential-time complexity, but it is generally polynomial-time or better in practice. It is an improvement over the algorithm to test all BFS of the convex feasible region and then choose the optimal BFS. It does this by arbitrarily selecting an initial vertex and then iteratively moving to an adjacent vertex, such that the objective function is improved. This algorithm still guarantees that the optimal point will be discovered ${ }^{9}$ and only in the worst-case scenario will all vertices be tested. A complete treatment of the Simplex algorithm is unnecessary here considering the scope of this paper; for a more complete treatment refer to any LP text such as Dantzig (1997); Hadley (1969). However, it is important to note that the revised Simplex algorithm, first proposed in 1953, is preferred to the initial Simplex algorithm. The advantages of the revised Simplex algorithm are listed by Dantzig (1997), the creator of the Simplex algorithm.

\footnotetext{
${ }^{8}$ A region is convex if the line between any two points in the region is fully contained within the region.

${ }^{9}$ In the case of an optimal point not existing, the algorithm will determine that the problem is infeasible.
} 


\section{A.6 Cutting Plane Algorithms}

Along with the Branch and Bound technique ${ }^{10}$, cutting plane algorithms are commonly used to solve discrete optimization problems. In the case of LP, cutting plane algorithms can be used to solve LP problems limiting variables to integer values, known as integer linear programming (ILP) problems. Cutting plane algorithms have been extremely successful in solving ILP problems.

Cutting plane algorithms deal with discrete optimization problems without the discrete variable limitation. The search area of the continuous variable problem is continually refined until the solution is also a discrete variable solution: this solution is the optimal discrete solution. As the name suggests, the method used for refinement is adding new constraints (termed cutting planes) to the continuous variable problem that cut away some of the feasible region. The way these new constraints are formed is a non-trivial implementation issue; there are various ways, often problem-specific, and consequently there are numerous cutting plane algorithms. Furthermore, the algorithm will still take exponential-time in the worst-case scenario.

${ }^{10}$ Branch and Bound is out of scope of this paper, but most linear programming or operations research books will discuss this simple technique. 


\section{Appendix B - SDP Formulations of Selected References}

Leibfritz, F., and J. H. Maruhn, 2009, A successive SDP-NSDP approach to a robust optimization problem in finance, Computational Optimization and Applications 44(3), 443-466.

Leibfritz and Maruhn (2009) set up the following SDP formulation to boost the robustness of a previously published trading strategy. In formulating the optimization problem, they set up the financial instrument as a barrier option (up-and-out call), noting that if the stock price $\left(S_{t}\right)_{0 \leq t \leq T}$ hits the barrier $D\left(0<S_{0}<D\right)$ at some time $t \in[0, T]$ (knock-out), the option expires without value. Denoting the financial instruments in the hedge portfolio as $C_{1}, \ldots, C_{n}$ with values $C_{i}\left(t, S_{t}\right)$ at time $t$, then the units invested in product $C_{i}$ are $\alpha_{i}$, and the value of the hedge portfolio at time $t$ will be given by:

$$
\Pi(t, \alpha)=\sum_{i=1}^{n} \alpha_{i} C_{i}\left(t, S_{t}\right) .
$$

Hence the optimization problem of finding the cheapest trading strategy guaranteeing a payoff greater than or equal to the up-and-out call in all states of the economy is given by (Leibfritz and Maruhn, 2009):

$$
\min _{\alpha \in \mathbb{R}} \sum_{i=1}^{n} \alpha_{i} C_{i}\left(0, S_{0}\right)
$$

s.t. $\sum_{i: T_{i} \geq t} \alpha_{i} C_{i}(t, D) \geq 0$ for all times t the barrier might be hit,

$$
\sum_{i: T_{i}=T} \alpha_{i} \max \left(S_{T}-K_{i}, 0\right) \geq \max \left(S_{T}-K, 0\right) \quad \text { if the barrier is not hit at all. }
$$

Ye, Kai, P. Parpas, and B. Rustem, 2012, Robust portfolio optimization: a conic programming approach, Computational Optimization and Applications 52(2), 463-481.

The MMV model is well known to be sensitive to the mean and covariance of the returns, resulting in numerical instability when fund managers attempt to optimize their portfolios. Ye, Parpas and Rustem (2012) establish an SDP formulation of this problem which is robust to these input parameters. They establish the robust portfolio optimization problem as the following SDP problem:

$$
\begin{gathered}
\min _{\mathrm{X}, \mathrm{X}}\left\langle A_{0}, X\right\rangle+2 b_{0}^{T} x+c_{0} \\
\text { s.t. }\left\langle A_{1}, X\right\rangle+2 b_{1}^{T} x+c_{1} \leq 0 \\
{\left[\begin{array}{ll}
X & x \\
x & 1
\end{array}\right] \geqslant 0}
\end{gathered}
$$

where,

$$
\bar{X}=\left[\begin{array}{cc}
\hat{x} \hat{x}^{T} & \hat{x} \\
\hat{x}^{T} & 1
\end{array}\right]
$$

Here, they use the following portfolio quadratic matrix variable for the above matrix $\bar{X}$, where $w \in W$ are the weights of a portfolio. 


$$
\widehat{W}=\left[\begin{array}{cc}
w w^{T} & w \\
w^{T} & 1
\end{array}\right]
$$

The interested reader is referred to their paper for the specification of the symmetric matrices $A_{i}, i=0,1$ and $b_{i} \in \mathbb{R}^{n}, c_{i} \in \mathbb{R}$.

Lobo, M. S., M. Fazel, and S. Boyd, 2007, Portfolio optimization with linear and fixed transaction costs, Annals of Operations Research 152(1), 341-365.

As a specialized subset of SDP, it is interesting to note that a second-order cone problem formulation of the base problem of optimizing portfolios that are linear and have fixed transactions cost is well explain by Lobo, Fazel and Boyd (2007), wherein they formulate the portfolio selection problem as:

$$
\begin{array}{ll}
\text { Maximize: } & \bar{a}^{T}(w+x) \\
\text { Subject to } & \mathbf{1}^{T} x+\phi(x) \leq 0 \\
w+x \in \mathcal{S} &
\end{array}
$$

Where

$\bar{a} \in \mathbf{R}^{n} \quad$ is the vector of expected returns on each asset,

$w \in \mathbf{R}^{n}$ is the vector of current holdings in each asset,

$x \in \mathbf{R}^{n} \quad$ is the vector of amounts transacted in each asset,

$\phi: \mathbf{R}^{n} \rightarrow \mathbf{R} \quad$ is the transaction cost function,

$\mathcal{S} \subseteq \mathbf{R}^{n}$ is the set of feasible portfolios.

They note that the related problem of minimizing the transaction cost subject to the portfolio constraints is written as the following associated minimization problem:

Minimize $\phi(x)$

Subject to $\bar{a}^{T}(w+x) \geq r_{\min }$

$w+x \in \mathcal{S}$

Where $r_{\min }$ is the desired lower bound on the returns.

Interestingly enough, they assume that transaction costs are linearly separable, allowing the sum of transaction costs per trade to be written as:

$$
\phi(x)=\sum_{i=1}^{n} \phi_{i}\left(x_{i}\right)
$$

Where $\phi_{i}$ is the transaction cost function for asset $i$. 


\section{Table}

Table 1

Top publications by citations according to the Scopus database.

\begin{tabular}{llll}
\hline Authors (Year) & Research Stream & Journal Category & Citations (per year) \\
\hline Goldfarb \& Iyengar (2003) & Portfolio Optimization & Operations Research & $316(22.6)$ \\
El Ghaoui, Oks \& Oustry (2003) & Option Pricing & Operations Research & $225(16.1)$ \\
Lobo, Fazel, Boyd (2007) & Portfolio Optimization & Operations Research & $105(10.5)$ \\
\hline
\end{tabular}

